\title{
Kasuistiken
}

Anaesthesist 2021 · 70:308-315

https://doi.org/10.1007/s00101-020-00864-w

Eingegangen: 13. Januar 2020

Überarbeitet: 20. August 2020

Angenommen: 4. September 2020

Online publiziert: 1. Oktober 2020

() Der/die Autor(en) 2020

\section{Zusatzmaterial online}

Die Online-Version dieses Beitrags (https:// doi.org/10.1007/s00101-020-00864w) enthält eine Stellungnahme des Krankenhausinformationsdienstes zum fremdblutfreien Behandlungsmanagement von Jehovas Zeugen.

Beitrag und Zusatzmaterial stehen Ihnen auf www.springermedizin.de zur Verfügung. Bitte geben Sie dort den Beitragstitel in die Suche ein, das Zusatzmaterial finden Sie beim Beitrag unter „Ergänzende Inhalte“.

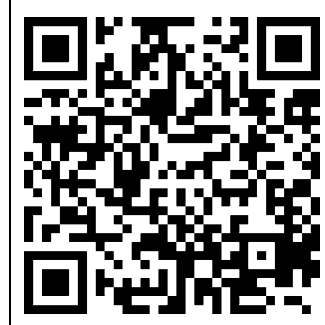

\section{Anamnese}

Eine 29-jährige, $172 \mathrm{~cm}$ große und $93 \mathrm{~kg}$ wiegende Zeugin Jehovas (ZJ) begab sich wegen vaginaler Blutungen in die stationäre Behandlung. Sie war in der 25 . Woche schwanger, Primipara, Secundigravida. Bei intrauterinem Fruchttod wurden eine Geburtseinleitung und bei fortgesetzter Blutung eine Nachkürettage durchgeführt. Prä- und postoperativ wurde sie von Anästhesisten und Gynäkologen auf die medizinische Notwendigkeit einer Bluttransfusion hingewiesen, um einer Gefahr für Leib und Leben vorzubeugen. Aufgrund ihres Glaubens lehnte die Patientin die Gabe

\author{
A. Meiser ${ }^{1}$ H. Knoll ${ }^{2} \cdot$ T. Meisel ${ }^{1}$ M. Schröder ${ }^{1} \cdot$ T. Volk $^{1}$ \\ ${ }^{1}$ Interdisziplinäre Operative Intensivstation, Klinik für Anästhesiologie, Intensivmedizin und \\ Schmerztherapie, Universitätsklinikum des Saarlandes, Homburg/Saar, Deutschland \\ ${ }^{2}$ Klinik für Allgemeine Chirurgie, Viszeral-, Gefäß- und Kinderchirurgie, Universitätsklinikum des \\ Saarlandes, Homburg/Saar, Deutschland
}

\section{Therapie einer extremen Anämie mit vernetztem Rinderhämoglobin}

\section{Fallbericht mit Literaturüberblick}

von Fremdblut sowie jeglichen anderen Blutprodukten ab. Etwa $18 \mathrm{~h}$ nach der Kürettage wurde die Patientin wegen $\mathrm{Ab}$ falls des Hämoglobins ( $\mathrm{Hb}$ ) auf 4,3 g/dl auf die Intensivstation aufgenommen. Bis dahin waren alle Vital- und Laborparameter bis auf $\mathrm{Hb}$ und Leukozyten unauffällig gewesen. Übelkeit, Kopf- und Oberbauchschmerzen wurden verneint. Vorerkrankungen waren nicht bekannt.

\section{Befund}

Bei Aufnahme zeigte sich eine wache, voll orientierte, in Ruhe kreislaufstabile Patientin ohne Blutungszeichen. Weder klinisch noch sonographisch gab es Hinweise auf eine fortgesetzte Blutung; laborchemisch zeigten sich Zeichen einer akuten Hämolyse, Erhöhungen der Transaminasen sowie eine Thrombozytopenie (- Tab. 1). Die Patientin war anurisch, Fragmentozyten waren nicht nachweisbar; Blutkulturen und Vaginalabstriche zeigten kein Keimwachstum; im Abdomen-CT konnten weder Blutungszeichen noch Perfusionsstörungen noch Verhalte nachgewiesen werden.

\section{Diagnose}

Aufgrund dieser Befunde gingen wir von einer Anämie nach Totgeburt mit vaginaler Blutung sowie einem postpartalen $\operatorname{HELLP}($ „hemolysis, elevated liver enzymes, low platelets“)-Syndrom mit Hämolyse, Leberenzymanstieg und Thrombozytenabfall aus. Als Ursache des Nieren- versagens kamen zum einen die blutungsbedingte Anämie, die Hämolyse, oder eine septische Ursache bei deutlich erhöhten Entzündungsparametern infrage.

\section{Therapie}

Trotz ausreichender Volumengabe blieb die Patientin anurisch. Nach ausführlicher Aufklärung der Patientin und der Angehörigen wurde eine Hämodialyse zur Therapie des Nierenversagens akzeptiert und als kontinuierliches Verfahren mit einer Zitratantikoagulation ab dem 3. Tag auf der Intensivstation (Tag 3) etabliert.

Alle Empfehlungen zum Patient Blood Management wurden umgesetzt (- Infobox 1) [17]. Es erfolgten eine Gerinnungsoptimierung mit Tranexamsäure und Desmopressin sowie eine Behandlung der Hämolyse mit Prednisolon. Zur Stimulation der Blutbildung wurden 3-mal wöchentlich Erythropoetin, anfangs 20.000 IE, zusammen mit einmalig $1000 \mathrm{mg}$ Eisen, sowie täglich $1000 \mu \mathrm{g}$ Cobalamin und $5 \mathrm{mg}$ Folsäure i.v. verabreicht. Durch Atemphysiotherapie und Sauerstoffinsufflation wurde die Sauerstoffsättigung optimiert. Blutentnahmen wurden reduziert und pädiatrische Monovetten verwendet. Die Dialysebehandlung wurde unter strikter Antikoagulation mit Zitrat durchgeführt, um ein Stehenbleiben des Systems auf jeden Fall zu vermeiden. Beim Systemwechsel der Dialyse wurde darauf geachtet, jeweils das gesamte Blutvolu- 


\begin{tabular}{|c|c|c|c|c|c|c|c|}
\hline & Prä-OP & Tag 1 & Tag 10 & Tag 12 & Tag 14 & Tag 20 & Tag 38 \\
\hline Blutdruck [mm Hg] & $140 / 80$ & $142 / 68$ & $131 / 60$ & $121 / 66$ & $150 / 70$ & $160 / 85$ & $143 / 80$ \\
\hline Herzfrequenz [/min] & 90 & 106 & 136 & 107 & 91 & 116 & 85 \\
\hline Temperatur $\left[{ }^{\circ} \mathrm{C}\right]$ & - & 37,4 & 37 & 36,8 & 37,4 & 38,2 & 37,4 \\
\hline Hämoglobin [g/dl] & 8,1 & 4,6 & 1,9 & 2,3 & 3,3 & 4,9 & 5,6 \\
\hline Met-Hb (\%) & - & - & - & 18 & 28 & 7 & - \\
\hline $\begin{array}{l}\text { Thrombozyten } \\
{\left[10^{9} / I\right]}\end{array}$ & 219 & 84 & 267 & 293 & 305 & 300 & 291 \\
\hline Quick [\%] & 102 & 77 & 73 & 65 & 78 & 81 & 80 \\
\hline $\operatorname{PTT}[s]$ & 19 & 28 & 29 & 24 & 18 & 32 & 18 \\
\hline Fibrinogen [mg/dl] & 312 & 232 & 296 & 315 & 361 & 549 & 253 \\
\hline Haptoglobin [mg/dl] & 123 & $<5$ & $<5$ & $<5$ & 6 & $<5$ & 37 \\
\hline$A S A T[U / I]$ & 19 & 550 & 42 & 82 & 132 & 63 & 19 \\
\hline$A L A T[U / I]$ & 10 & 200 & 19 & 22 & 27 & 15 & 24 \\
\hline$G L D H[U / I]$ & - & 520 & 21 & 21 & 22 & 6 & 2 \\
\hline$L D H[U / I]$ & 231 & 2600 & 1514 & 1243 & 1281 & 902 & 424 \\
\hline Kreatinin [mg/dl] & 0,48 & 3,3 & (CVVHD) & (CVVHD) & (CVVHD) & (CVVHD) & (CVVHD) \\
\hline Leukozyten $\left[10^{9} / I\right]$ & 15,5 & 15 & 13 & 22 & 19 & 11 & 9 \\
\hline$C R P[\mathrm{mg} / \mathrm{l}]$ & 10 & 150 & 70 & 63 & 115 & 185 & 3 \\
\hline$P C T[\mathrm{ng} / \mathrm{ml}]$ & - & 5,5 & 1,6 & 1,4 & 1,6 & 1,6 & 0,25 \\
\hline \multicolumn{8}{|c|}{$\begin{array}{l}\text { Die präoperativen Parameter (Prä-OP) wurden nach dem Abort und vor der Nachkürettage, die } \\
\text { Parameter am Tag } 1 \text { etwa } 18 \mathrm{~h} \text { später bei Aufnahme auf die Intensivstation erhoben } \\
\text { Met-Hb Methämoglobin (prozentualer Anteil am Gesamthämoglobin), } P T T \text { „partial thromboplastin } \\
\text { time", } A S A T \text { Aspartataminotransferase, ALAT Alaninaminotransferase, GLDH Glutamatdehydrogen- } \\
\text { ase, } L D H \text { Laktatdehydrogenase, CRP C-reaktives Protein, PCT Prokalzitonin, CVVHD Dialysebehand- } \\
\text { lung ("continuous venovenous haemodialysis") }\end{array}$} \\
\hline
\end{tabular}

men zurückzugeben. Dennoch fiel der Hb-Wert kontinuierlich bis auf $1,8 \mathrm{~g} / \mathrm{dl}$ am Tag 11 ab (• Abb. 1).

\section{Der klinische Verlauf}

Die Patientin wurde täglich in den Stand mobilisiert. Am Tag 8 ( $\mathrm{Hb} 2,8 \mathrm{~g} / \mathrm{dl}$ ) gelang die Mobilisation nur bis an die Bettkante und musste wegen einer Tachykardie abgebrochen werden. Am Tag $10(\mathrm{Hb}$ $1,9 \mathrm{~g} / \mathrm{dl}$ ) wurde die Patientin während der ärztlichen Untersuchung in Rückenlage plötzlich bewusstlos und musste schutzintubiert werden. Zur Reduktion des Sauerstoffverbrauchs wurde die Patientin inhalativ mit Isofluran sediert. Wiederholtes Shivering, Pressen gegen die Beatmung und Strecksynergismen wurden als Krampanfälle gedeutet und mit Levetiracetam und Vertiefung der Sedie-

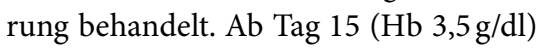
atmete die Patientin spontan mit Druck-

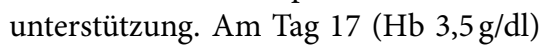
wurden im Sedierungsfenster krampftypische Potenziale im EEG nachgewiesen,

\section{Polymerisiertes bovines Hämoglobin als Blutersatz (Hemopure ${ }^{\circledR}$ )}

Nach dem Bewusstseinsverlust am Tag 10 trotz Ausschöpfung aller Therapiemaßnahmen gingen wir von einer akuten $\mathrm{Ge}$ fährdung der jungen Mutter aus. In wiederholten Gesprächen mit Mitgliedern der Glaubensgemeinschaft, dem vorsorgebevollmächtigten Ehemann und der Patientin auch unter vier Augen war die Gabe von Fremdblut stets abgelehnt worden. Gemeinsam mit der Glaubensgemeinschaft wurde diskutiert, das Blutersatzpräparat Hemopure ${ }^{\circledR}$ (Fa. Hemoglobin Oxygen Therapeutics LLC, Souderton, PA, USA) einzusetzen, welches in keinem Land der europäischen Union zugelassen ist.

Hemopure $^{\circledR}$ besteht aus vernetztem Rinder-Hb. Das Polymer mit einer molaren Masse von $250 \mathrm{~kg} / \mathrm{mol}$ ist in $250 \mathrm{ml}$ Ringer-Lösung suspendiert. Ein Infusionsbeutel enthält $32 \mathrm{~g} \mathrm{Hb}$ mit einer Sauerstoffbindungskapazität von $1,39 \mathrm{ml}$ $\mathrm{O}_{2} / \mathrm{g} \mathrm{Hb}$, analog der des menschlichen Hb. Durch eine Rechtsverschiebung der Sauerstoffbindungskurve ist die Sauerstoffabgabe an die Gewebe verbessert, die Sauerstoffaufnahme in der Lunge bei hohen Sauerstoffkonzentrationen jedoch nicht verschlechtert. Die Lösung zeigt kolloidale Eigenschaften, sodass die Gefahr einer Volumenüberladung besteht. Da es sich um eine zellfreie Lösung handelt, ist das immunisierende Potenzial gering, eine serologische Verträglichkeitsprobe nicht durchführbar. Das polymerisierte $\mathrm{Hb}$ wird in der Niere nicht filtriert und vom retikuloendothelialen System rasch abgebaut, sodass die Gabe täglich wiederholt werden muss. Es ist nicht wie das körpereigene $\mathrm{Hb}$ durch die enzymatische Ausstattung der Erythrozyten vor Oxidation geschützt und neigt daher zur Bildung von Methämoglobin (Met-Hb), welches für den Sauerstofftransport nicht mehr zur Verfügung steht. Die zeitgleiche Infusion von Ascorbinsäure als Antioxidans wird daher empfohlen. Hemopure ist ohne Kühlung 3 Jahre haltbar, wird in den USA hergestellt und ist in Russland und Südafrika als Blutersatzmittel zugelassen [16]. 
Anaesthesist 2021 · 70:308-315 https://doi.org/10.1007/s00101-020-00864-w

(c) Der/die Autor(en) 2020

\section{A. Meiser · H. Knoll · T. Meisel · M. Schröder · T. Volk \\ Therapie einer extremen Anämie mit vernetztem Rinderhämoglobin. Fallbericht mit Literaturüberblick}

\section{Zusammenfassung}

Eine 29-jährige Zeugin Jehovas erlitt nach intrauterinem Fruchttod in der 25. Schwangerschaftswoche ein akutes Nierenversagen und eine schwere Anämie, bedingt durch Blutverlust und Hämolyse, erlitt. Wegen ihrer religiösen Überzeugung lehnte die Patientin Bluttransfusionen kategorisch ab. Trotz Umsetzung aller Empfehlungen des Patient Blood Management fiel der Hämoglobin(Hb)-Wert kontinuierlich. Am Tag 10 wurde sie bei $\mathrm{Hb}$ 1,9 g/dl plötzlich bewusstlos und musste intubiert und beatmet werden. Wegen organprotektiver Effekte und der guten Steuerbarkeit wurde sie inhalativ mit Isofluran sediert. Isofluran ermöglichte jeweils eine rasche neurologische Beurteilbarkeit in Sedierungsfenstern sowie eine regelmäßige, ruhige Spontanatmung bei tiefer Sedierung mit reduziertem Sauerstoffverbrauch. Als Ausdruck einer posthypoxischen Enzephalopathie zeigte sie in den Sedierungsfenstern Krampfanfälle, die im EEG bestätigt und antikonvulsiv behandelt wurden. Bei $\mathrm{Hb}$ 1,8 $\mathrm{g} / \mathrm{dl}$ erhielt sie 2 Infusionsbeutel mit polymerisiertem bovinem Hämoglobin (Hemopure ${ }^{\circledR}$, Fa. Hemoglobin Oxygen Therapeutics LLC, Souderton, PA, USA), wegen der kurzen Halbwertszeit mehrmals wiederholt an den Folgetagen. Eine beachtliche Methämoglobinämie wurde festgestellt. Auch nach Abzug des Met- $\mathrm{Hb}$ zeigten sich $\mathrm{Hb}$-Anstiege um 0,4-0,8 g/dl nach den Gaben. Die Sauerstoffbindungskapazität des Blutes konnte damit initial um $33 \%$ gesteigert werden. Es kam zu einer vollständigen neurologischen Erholung, und die Patientin wurde schließlich vom Respirator entwöhnt, jedoch weiterhin dialysepflichtig nach 38 Tagen in eine andere Klink verlegt. Wenn die Gabe von Fremdblut keine Option darstellt, kann durch Gabe von polymerisiertem bovinem Hämoglobin als Ultima-Ratio-Therapie die Sauerstofftransportkapazität vorübergehend erhöht werden. Eine inhalative Sedierung mit Isofluran zur Senkung des Sauerstoffverbrauchs erscheint gleichermaßen vorteilhaft.

\section{Schlüsselwörter}

Polymerisiertes bovines Hämoglobin . Methämoglobin · Inhalative Sedierung · Isofluran

\section{Treatment of extreme anemia with polymerized bovine hemoglobin. Case report and review of the literature}

\begin{abstract}
This article reports the case of a 29-year-old female Jehovah's Witness with severe anemia after intrauterine fetal death in the 25th week of gestation, complicated by vaginal bleeding, acute renal failure and hemolysis. Due to her religious beliefs the patient categorically refused blood transfusions. Despite adhering to the recommendations for patient blood management, the hemoglobin $(\mathrm{Hb})$ level gradually decreased to $1.9 \mathrm{~g} / \mathrm{dl}$ on day 10 , when she fainted and had to be sedated and invasively ventilated. Inhalative isoflurane was chosen for sedation because of its potential organ-protective effects and because it provides deep sedation with reduced oxygen requirements, while enabling
\end{abstract}

rapid neurological examination during the sedation windows as well as regular and calm spontaneous breathing. Posthypoxic encephalopathy was demonstrated clinically and electroencephalographically by seizure activity during the sedation windows. Anticonvulsive treatment was started. At a hemoglobin of $1.8 \mathrm{~g} / \mathrm{dl}$, she received 2 units of polymerized bovine hemoglobin (Hemopure ${ }^{\circledR}$, Hemoglogin Oxygen Therapeutics LLC, Souderton, PA, USA), repeated several times on subsequent days because of its short half-life. Considerable methemoglobinemia was noted. After subtracting methemoglobin, the hemoglobin rose by $0.4-0.8 \mathrm{~g} / \mathrm{dl}$ after each 2 units, initially increasing the oxygen binding capacity of the blood by $33 \%$. After a full neurological recovery and weaning from the ventilator but still on hemodialysis, the patient was transferred to another hospital after 38 days.

If allogeneic blood transfusion is not an option, administration of polymerized bovine hemoglobin can temporarily increase the oxygen transport capacity as a last resort treatment. Reduction of oxygen requirements by deep inhaled sedation with isoflurane also seems beneficial and provides advantages.

Keywords

Polymerized bovine hemoglobin .

Methemoglobin · Inhaled sedation · Isoflurane
Der bevollmächtigte Ehemann wurde über die Anwendung des nichtzugelassenen Arzneimittels als individuellen Heilversuch aufgeklärt und um schriftliches Einverständnis gebeten. Hemopure ${ }^{\circledR}$ wurde bei der Firma bestellt und sollte aus den USA oder aus Südafrika geliefert werden. Dies hätte jedoch 7 bis 10 Tage in Anspruch genommen. In der Notfallsituation wurde ein Vorrat an Studienmedikation aus dem benachbarten Ausland vom Hersteller und vom Studienzentrum freigegeben und über Nacht geliefert.

An den Tagen 11 bis 16 und am Tag 18 wurden jeweils 2 Infusionsbeutel à $250 \mathrm{ml}$ über je $2 \mathrm{~h}$ nacheinander infundiert. $\mathrm{Zu}$ vor und danach wurden $\mathrm{Hb}$ und MetHb der Patientin bestimmt (• Abb. 1). Trotz Gabe von Ascorbinsäure [18] und Methylenblau [5] ließ sich die Bildung von Met-Hb bis über $20 \%$ des Gesamt$\mathrm{Hb}$ nicht verhindern. Hierunter war die pulsoxymetrische Sättigung klinisch nicht mehr verwertbar und zeigte Werte unter $80 \%$ an. Auch wenn man das Met$\mathrm{Hb}$, welches keinen Sauerstoff transportieren kann, vom Gesamt-Hb abzieht, kam es zu einem messbaren Hb-Anstieg unter der Therapie, der nicht durch patienteneigenes $\mathrm{Hb}$ erklärt werden kann, da der Hämatokrit vom Tag 10 bis Tag 13 gleichblieb und auch danach nur sehr viel langsamer anstieg. Klinisch zeigte sich ein Sklerenikterus, welcher durch Ablagerungen von Hemopure ${ }^{\circledR}$-Abbau- 


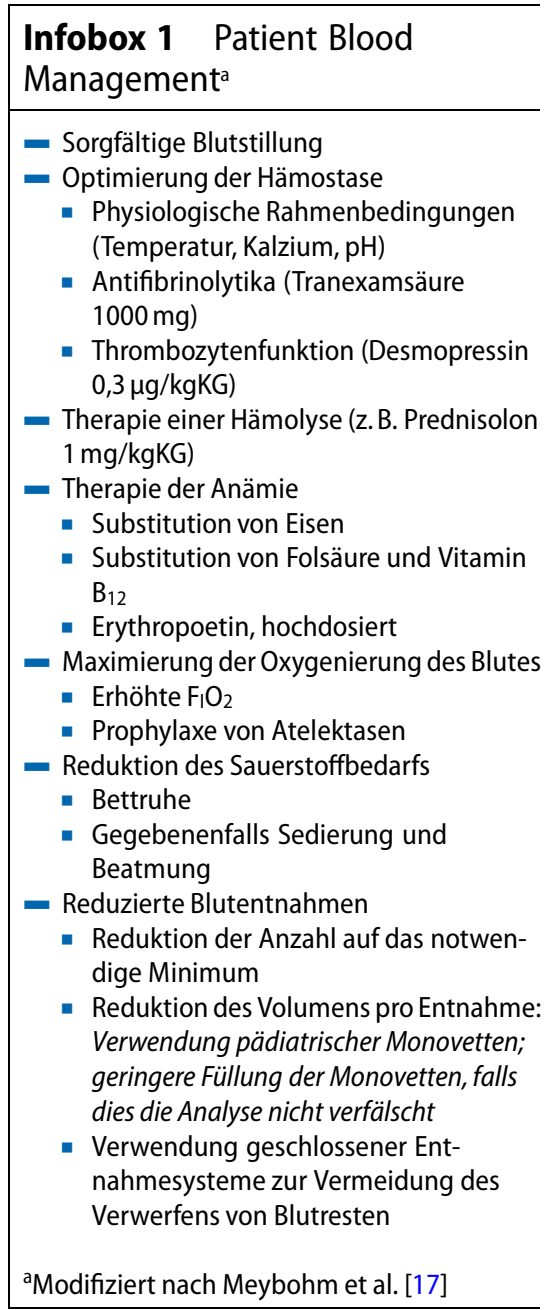

produkten erklärbar ist (• Abb. 2). Das Gesamtbilirubin stieg unter der Therapie bis maximal 2,9 mg/dl an Tag 15.

\section{Diskussion}

Wir berichten den Fall einer jungen Zeugin Jehovas, die einen kontinuierlichen Hb-Abfall bis auf 1,8 g/dl ohne neurologische Schäden überlebt hat. Entscheidende Therapiemaßnahmen waren neben Stimulation der Erythropoese und Behandlung der Hämolyse die Senkung des Sauerstoffverbrauchs durch tiefe, inhalative Sedierung mit Isofluran sowie die Gabe des Blutersatzstoffes Hemopure ${ }^{\circledR}$.

Etliche Fallberichte beschreiben eine erfolgreiche Therapie extrem niedriger $\mathrm{Hb}$-Werte bei jungen (maximales Alter 44 Jahre), kardiovaskulär gesunden ZJ, einige davon aus Europa [4, 21, 22], die meisten aus den USA $[5,7,10,20,23,24$, 28]. Sowohl akute postpartale [21, 23, 24], postoperative $[7,22,28]$ oder posttraumatische Anämien [20] als auch langsame Hb-Abfälle bei hämolytischer Anämie [10] oder Chemotherapie bei Leukämien $[4,5]$ wurden überlebt. Die niedrigsten dokumentierten $\mathrm{Hb}$-Werte reichen von 3,9 bis $1,3 \mathrm{mg} / \mathrm{dl}$ [4] sowie in einem Fall „nicht mehr messbar“ bei einem Hämatokrit von $3 \%$ [23].

In fast allen Fällen wurde dabei hochdosiertes Erythropoetin eingesetzt, aber nicht immer konnte damit eine rasche Erythropoese erzielt werden. Als Gründe werden Infekte, Nebenwirkung der Chemotherapie, aber auch die anämiebedingte Hypoxie des Knochenmarks selbst angeführt. In einem Fall konnten Kreislaufparameter (Laktacidose, zentralvenöse Sättigung und Herzfrequenz) durch Erhöhung der $\mathrm{F}_{\mathrm{I}} \mathrm{O}_{2}$ auf $100 \%$ verbessert werden [23], in einem anderen Fall zeigten wiederholte Zyklen einer hyperbaren Sauerstofftherapie keinen messbaren Effekt [24].

In den USA wurden in 5 publizierten Fällen 2 bis 15 Infusionsbeutel Hemopure $^{\circledR}$ eingesetzt $[5,7,10,20$, 24]. Die Autoren beschreiben messbare Hb-Anstiege, aber auch eine Erhöhung des Met-Hb. Über die Anwendung von Hemopure ${ }^{\circledR}$ in Ländern der Europäischen Union wurde bislang nicht berichtet.

Fallberichte zeigen einen Publikationsbias, da oftmals nur ein gutes Outcome publiziert wird. Das Mortalitätsund Morbiditätsrisiko extremer Anämien kann nur durch systematische Analysen abgeschätzt werden. Im Rahmen einer retrospektiven Analyse wurde von einem postpartalen Todesfall einer 40jährigen $\mathrm{ZJ}$ mit einem $\mathrm{Hb}$ von $3,4 \mathrm{~g} / \mathrm{dl}$ berichtet [12]. Bei einer nationalen Registeranalyse in den Niederlanden wurden 6 postpartal verstorbene ZJ auf $8850 \mathrm{Ge}$ burten identifiziert, welches einer 6-fach erhöhten Müttersterblichkeit entspricht [27]. Die Mortalität von 300 Patienten, die Bluttransfusionen ablehnten, in den USA an spezialisierten Zentren operiert wurden und die postoperative $\mathrm{Hb}$-Werte kleiner $8 \mathrm{~g} / \mathrm{dl}$ aufwiesen, korrelierte mit dem niedrigsten gemessenen Hb-Wert. Sie betrug $0 \%$ für $\mathrm{Hb}$-Werte $7-8 \mathrm{~g} / \mathrm{dl}$ und stieg bis auf $54 \%$ und $100 \%$ für $\mathrm{Hb}$-Werte 2-3 sowie $1-2 \mathrm{~g} / \mathrm{dl}[2]$.
In den meisten Fallberichten wurden Patienten mit extremer Anämie sediert und beatmet. Wir verwenden für die tiefe Sedierung Isofluran, verabreicht über das AnaConDa ${ }^{\circledR}$-System (Fa. Sedana Medical, Danderyd, Schweden). Hierbei handelt es sich um einen „off label use“, der jedoch durch zahlreiche randomisierte Studien (reviewed in $[8,14]$ ) und mehrere nationale Leitlinien gedeckt ist $[1,3$, 26]. Die rasche neurologische Beurteilbarkeit [6] in Sedierungsfenstern erlaubte uns die Diagnose einer posthypoxischen Enzephalopathie durch Nachweis epileptiformer Potenziale im EEG sowie die Initiierung einer ruhigen Spontanatmung [13, 15], um Zwerchfellatrophie und Atelektasenbildung in den dorsobasalen Lungenabschnitten vorzubeugen, bei zugleich tiefer Sedierung mit reduziertem Sauerstoffverbrauch. Des Weiteren werden volatilen Anästhetika organprotektive Wirkungen zugeschrieben (reviewed in [9]).

In einer Metaanalyse von 16 Studien mit insgesamt 3711 Patienten, die verschiedene zellfreie hämoglobinbasierte Blutersatzstoffe mit der Gabe von Erythrozyten verglichen, zeigten sich eine höhere Mortalität und ein häufigeres Auftreten von Myokardinfarkten in den Studiengruppen [19]. Ursächlich ist vermutlich ein Abfangen von Stickstoffmonoxid als potentem körpereigenem Vasodilatator [11]. Aufgrund dieser Daten wurde keines der Präparate von der US-amerikanischen Food and Drug Administration (FDA) zugelassen. Wenn Erythrozytenkonzentrate jedoch keine Option darstellen, ist das Risiko, an einer extremen Anämie zu versterben, wesentlich höher als das gering erhöhte Mortalitätsrisiko durch Blutersatzstoffe [25].

Der plötzliche Bewusstseinsverlust und die nachgewiesenen Krampfpotenziale bei einer Patientin ohne Epilepsie in der Vorgeschichte beweisen, dass die Sauerstoffversorgung des Gehirns zeitweise kompromittiert war. Wir gehen daher davon aus, dass sich die Gabe von Hemopure $^{\circledR}$ auf den weiteren Verlauf positiv ausgewirkt hat, auch wenn die effektive, Met-Hb-bereinigte Hb-WertSteigerung nur $0,4-0,8 \mathrm{~g} / \mathrm{dl}$ ausmachte. Dies entsprach jedoch initial einer Stei- 


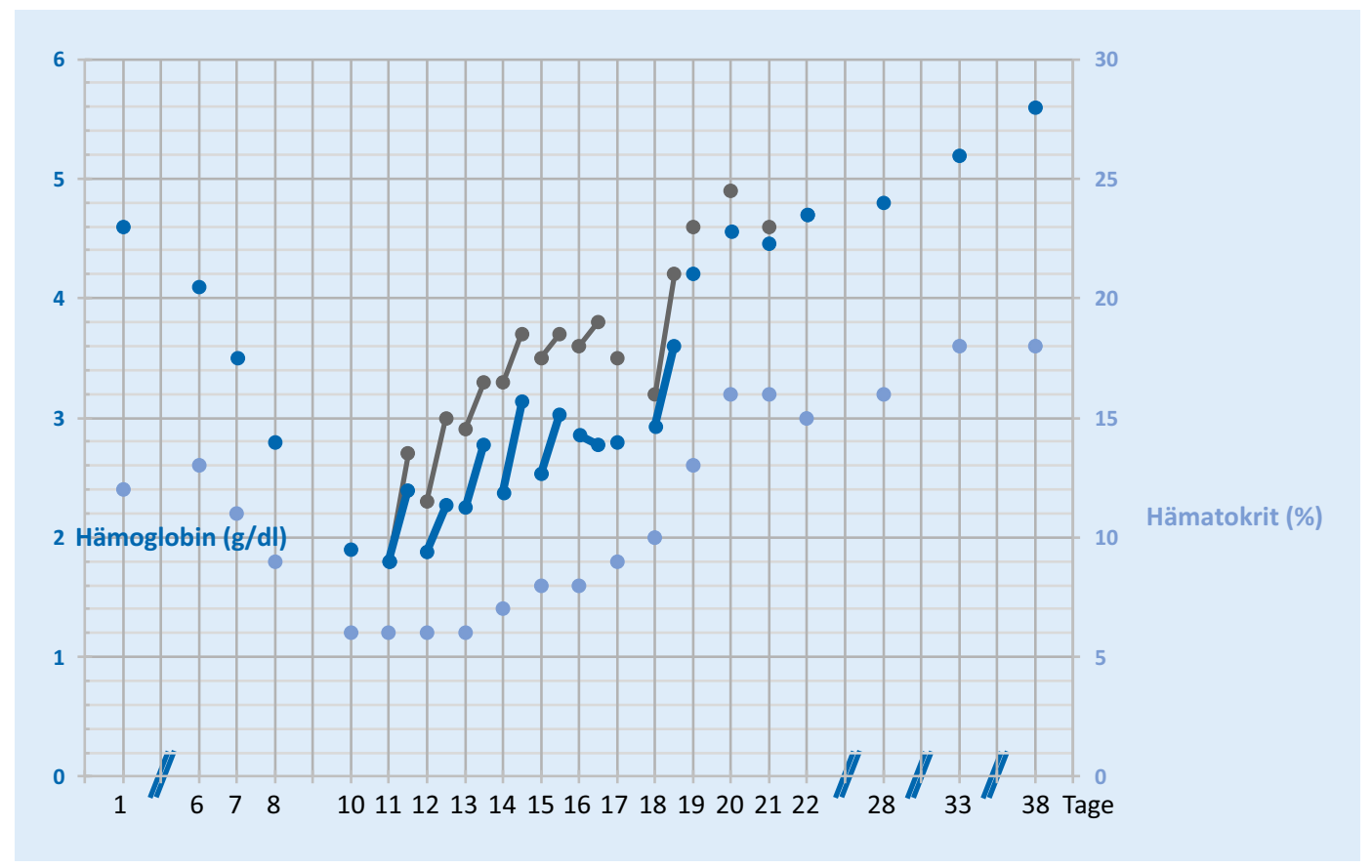

Abb. 1 ॥ Die Abbildung zeigt den Verlauf des Hämoglobins ( $\mathrm{Hb}$, blaue und graue Punkte, linke Skala) und des Hämatokrits (hellblaue Punkte, rechte Skala) während des Intensivaufenthalts. An den Tagen 11 bis 16 und 18 wurde polymerisiertes Rinder-Hb (Hemopure ${ }^{\circledR}$ ) verabreicht. Das zellfreie Präparat führt zu einem messbaren Anstieg des $\mathrm{Hb}$, nicht jedoch des Hämatokrits. Verbundene Punkte stellen $\mathrm{Hb}$-Werte unmittelbar vor und nach Hemopure ${ }^{\circledR}$-Gabe dar. Ein Teil des infundierten $\mathrm{Hb}$ wird im Körper zu Met-Hb oxidiert, welches keinen Sauerstoff binden kann. Die grauen Punkte stellen das gesamte Hb, die blauen nur den Anteil ohne Met-Hb dar
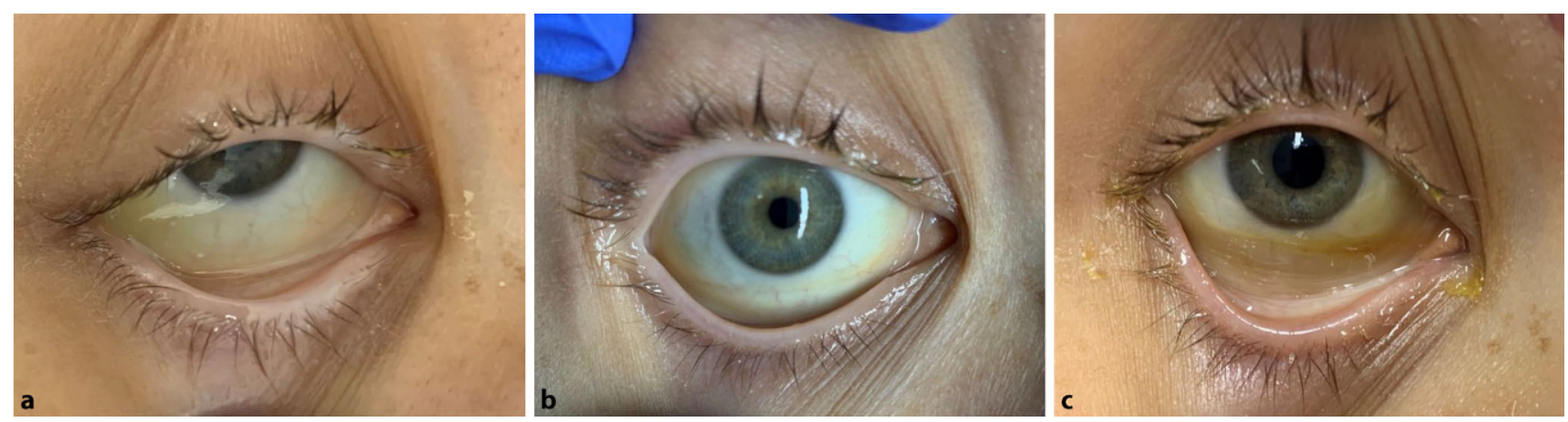

Abb. 2 \ Rechtes Auge der Patientin. Die Konjunktiven zeigen eine deutliche Blässe. a Tag 12, Hb 1,9 g/dl, beginnender Sklerenikterus nach 2 Einheiten Hemopure ${ }^{\circledR}$. b Tag 13, Hb 2,9 g/dl, Zunahme des Sklerenikterus nach 4 Einheiten Hemopure ${ }^{\circledR}$. c Tag 14, Hb 3,3 g/dl, deutlicher Sklerenikterus nach 6 Einheiten Hemopure ${ }^{\circledR}$

gerung der Sauerstoffbindungskapazität des Blutes um $33 \%$.

Bei einem $\mathrm{Hb}$ von $1,8 \mathrm{~g} / \mathrm{dl}$ und einem geschätzten Blutvolumen von 6,31 $(0,07$ * $90 \mathrm{kgKG})$ kann das Hämoglobin der Patientin etwa $158 \mathrm{ml} \mathrm{O}_{2}$ binden. Zusätzlich sind bei einem $\mathrm{p}_{\mathrm{a}} \mathrm{O}_{2}$ von $250 \mathrm{~mm}$ Hg etwa $50 \mathrm{ml} \mathrm{O}_{2}$ physikalisch gelöst. Eine Einheit Hämopure mit $32 \mathrm{~g} \mathrm{Hb}$ kann $44 \mathrm{ml}$ $\mathrm{O}_{2}$ binden. Durch 2 Einheiten des Präparats konnte also der Sauerstoffgehalt des gesamten Blutes um $42 \%$ auf $296 \mathrm{ml}$
$\mathrm{O}_{2}$ gesteigert werden. Allerdings muss erwähnt werden, dass ein Teil des zugeführten Hämoglobins trotz Gabe von Ascorbinsäure (und später zusätzlich Methylenblau) sehr rasch zu Met-Hb oxidierte und damit keinen Sauerstoff mehr transportieren konnte. In $\bullet \mathbf{A b b} .1$ ist daher der jeweilige Anstieg des Hb unter der täglich wiederholten Hemopure ${ }^{\circledR}$-Therapie sowohl als Gesamt-Hb wie auch als Anteil ohne Met-Hb dargestellt. Der rasche Abbau durch das retikuloendothe- liale System führte dazu, dass bereits am nächsten Morgen das Gesamt-Hb wieder von 2,7 auf 2,3 (Anteil ohne Met-Hb: von 2,5 auf 1,9) $\mathrm{g} / \mathrm{dl}$ abgefallen war.

Es ist einem Zufall zu verdanken, dass das Arzneimittel kurzfristig aus dem nahen Ausland besorgt werden konnte. Dies hat der Patientin wahrscheinlich das Leben gerettet. In den USA ist Ärzten die Verabreichung nichtzugelassener Medikamente als „compassionate use " nur erlaubt mit einer Genehmigung durch die 
FDA, die für ZJ bislang in 109 Fällen erteilt wurde (zitiert aus [25]). In Deutschland kann ein Arzt im Sinne eines Heilversuchs im Einzelfall nichtzugelassene Arzneimittel ohne behördliche Genehmigung unter Berufung auf einen rechtfertigenden Notstand einsetzen. Dabei trägt der Arzt die Verantwortung; er muss umfassend aufklären, ein Einverständnis einholen und die Behandlung sorgfältig dokumentieren [29]. Für einen wiederholten Einsatz bei mehreren Patienten mit der gleichen Indikation kann ein Arzneimittelhärtefallprogramm beim Bundesamt für Arzneimittel und Medizinprodukte beantragt werden [30]. Allerdings stellt die Beschaffung eines Medikaments aus dem Ausland letztlich eine zeitaufwendige Prozedur dar, da sowohl eine Ausfuhr- als auch eine Einfuhrgenehmigung beschafft werden müssen. Diese bürokratischen Hürden nehmen einige Tage bis Wochen in Anspruch, selbst wenn der Antrag als „medizinischer Notfall“ gekennzeichnet ist.

\section{Fazit für die Praxis}

Wenn die Gabe von Fremdblut aus medizinischen (Autoimmunhämolyse), logistischen (Verfügbarkeit) oder aus Glaubensgründen keine Option darstellt, kann durch Gabe von polymerisiertem bovinem Hämoglobin als Ultima-Ratio-Therapie die Sauerstofftransportkapazität vorübergehend erhöht werden. Wegen der kurzen Halbwertszeit sind wiederholte Gaben erforderlich, zumal eine rasche Methämoglobinbildung den Sauerstofftransport des zugeführten Hämoglobins kompromittiert. Eine Senkung des Sauerstoffverbrauchs ist gleichermaßen wichtig; eine inhalative Sedierung mit Isofluran stellt hier eine gute Option dar.

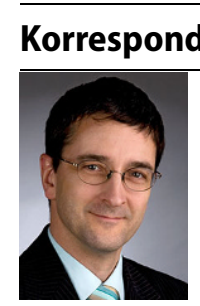

Interdisziplinäre Operative Intensivstation, Klinik für Anästhesiologie, Intensivmedizin und

Schmerztherapie, Universitätsklinikum des Saarlandes Kirrberger Str. 100, 66424 Homburg/Saar, Deutschland andreas.meiser@uks.eu

Funding. Open Access funding enabled and organized by Projekt DEAL.

\section{Einhaltung ethischer Richtlinien}

Interessenkonflikt. A. Meiser gibt an, als Berater für die Fa. Sedana Medical tätig zu sein und Honorare, Reisekosten und Vortragshonorare erhalten zu haben. A. Meiser hat keine Interessenkonflikte zu Blutersatzstoffen, Hemopure und in Bezug auf die Glaubensgemeinschaft der Zeugen Jehovas. T. Volk gibt an, "coordinating investigator " für eine klinische Prüfung der Fa. Sedana Medical zu sein. Er erhielt ein Beraterhonorar. Es bestehen keine Interessenkonflikte zu Blutersatzstoffen, Hemopure und in Bezug auf die Glaubensgemeinschaft der Zeugen Jehovas H. Knoll, T. Meisel und M. Schröder geben an, dass kein Interessenkonflikt besteht.

Für diesen Beitrag wurden von den Autoren keine Studien an Menschen oder Tieren durchgeführt. Für die aufgeführten Studien gelten die jeweils dort angegebenen ethischen Richtlinien. Für Bildmaterial oder anderweitige Angaben innerhalb des Manuskripts, über die Patienten zu identifizieren sind, liegt von ihnen und/oder ihren gesetzlichen Vertretern eine schriftliche Einwilligung vor.

Open Access. Dieser Artikel wird unter der Creative Commons Namensnennung 4.0 International Lizenz veröffentlicht, welche die Nutzung, Vervielfältigung, Bearbeitung, Verbreitung und Wiedergabe in jeglichem Medium und Format erlaubt, sofern Sie den/die ursprünglichen Autor(en) und die Quelle ordnungsgemäßnennen, einen Link zur Creative Commons Lizenz beifügen und angeben, ob Änderungen vorgenommen wurden.

Die in diesem Artikel enthaltenen Bilder und sonstiges Drittmaterial unterliegen ebenfalls der genannten Creative Commons Lizenz, sofern sich aus der Abbildungslegende nichts anderes ergibt. Sofern das betreffende Material nicht unter der genannten Creative Commons Lizenz steht und die betreffende Handlung nicht nach gesetzlichen Vorschriften erlaubt ist, ist für die oben aufgeführten Weiterverwendungen des Materials die Einwilligung des jeweiligen Rechteinhabers einzuholen.

Weitere Details zur Lizenz entnehmen Sie bitte der Lizenzinformation auf http://creativecommons.org/ licenses/by/4.0/deed.de.

\section{Literatur}

1. Baron R, Binder A, BiniekRetal (2015) Evidenceand consensus based guideline for the management of delirium, analgesia, and sedation in intensive care medicine. Revision 2015 (DAS-Guideline 2015)—short version. Ger Med Sci 13:1-42. https://doi.org/10.3205/000223

2. Carson JL, Noveck H, Berlin JA, Gould SA (2002) Mortality and morbidity in patients with very low postoperative $\mathrm{Hb}$ levels who decline blood transfusion. Transfusion 42:812-818. https://doi org/10.1046/j.1537-2995.2002.00123.x

3. Celis-Rodríguez $\mathrm{E}$, Birchenall $C$, de la Cal MÁ et al (2013) Guía de práctica clínica basada en la evidencia para el manejo de la sedoanalgesia en el paciente adulto críticamente enfermo. Med Intensiva 37:519-574. https://doi.org/10.1016/j. medin.2013.04.001

4. Chojnowski K, Janus A, Bliźniewska K et al (2016) Long-lasting extreme anemia during the therapy of acute lymphoblastic leukemia in a Jehovah's witness patient. Transfusion 56:2438-2442. https://doi.org/10.1111/trf.13703

5. Donahue LL, Shapira I, Shander A et al (2010) Management of acute anemia in a Jehovah's witness patient with acutelymphoblasticleukemia with polymerized bovine hemoglobin-based oxygen carrier: a case report and review of literature. Transfusion 50:1561-1567. https://doi. org/10.1111/j.1537-2995.2010.02603.x

6. Hellstrom J, Öwall A, Martling CR, Sackey PV (2014) Inhaled isoflurane sedation during therapeutic hypothermia after cardiac arrest: a case series. Crit Care Med 42:e161-e166

7. Henderson R, Chow JH, Tanaka KA (2019) A bridge to bloodless surgery: use of hemoglobinbased oxygen carrier for anemia treatment and autologous blood preservation during redo pulmonic valve replacement. J Cardiothorac Vasc Anesth 33:1973-1976. https://doi.org/10.1053/j. jvca.2018.09.036

8. Jerath A, Panckhurst J, Parotto M et al (2017) Safety and efficacy of volatile anesthetic agents compared with standard intravenous midazolam/ propofol sedation in ventilated critical care patients: A meta-analysis and systematic review of prospective trials. Anesth Analg 124:1190-1199. https://doi.org/10.1213/ANE.0000000000001634

9. Jerath $A$, Parotto $M$, Wasowicz $M$, Ferguson ND (2016) Volatile anesthetics. Is a new player emerging in critical care sedation? Am J Respir Crit Care Med 193:1202-1212. https://doi.org/10. 1164/rccm.201512-2435CP

10. Jordan SD, Alexander E (2013) Bovine hemoglobin: a nontraditional approach to the management of acute anemia in a Jehovah's witness patient with autoimmune hemolytic anemia. J Pharm Pract 26:257-260. https://doi.org/10. $1177 / 0897190012451928$

11. Kubulus D, Rensing H, Paxian M et al (2005) Influence of heme-based solutions on stress protein expression and organ failure after hemorrhagic shock. Crit Care Med 33:629-637. https://doi.org/ 10.1097/01.ccm.0000156295.48075.49

12. Massiah N, Athimulam S, Loo C et al (2007) Obstetric care of Jehovah's witnesses: a 14year observational study. Arch Gynecol Obstet 276:339-343. https://doi.org/10.1007/s00404007-0346-0

13. Meiser A, Bomberg $H$, Lepper PM et al (2017) Inhaled sedation in patients with acute respiratory distress syndrome undergoing extracorporeal membrane oxygenation. Anesth Analg 
125:1235-1239. https://doi.org/10.1213/ANE. 0000000000001915

14. Meiser A, Bomberg H, Volk T, Groesdonk HV (2017) Neue technische Entwicklungen der inhalativen Sedierung. Anaesthesist 66:274-282. https://doi. org/10.1007/s00101-017-0269-5

15. Meiser A, Groesdonk HV, Bonnekessel S et al (2018) Inhalation sedation in subjects with ARDS undergoing continuous lateral rotational therapy. Respir Care 63:441-447. https://doi.org/10.4187/ respcare.05751

16. Mer M,Hodgson E, Wallis Letal (2016) Hemoglobin glutamer-250 (bovine) in South Africa: consensus usage guidelines from clinician experts who have treated patients. Transfusion 56:2631-2636. https://doi.org/10.1111/trf.13726

17. Meybohm P, Schmitz-Rixen T, Steinbicker A et al (2017) Das Patient-Blood-Management-Konzept: Gemeinsame Empfehlung der Deutschen Gesellschaft für Anästhesiologie und Intensivmedizin und der Deutschen Gesellschaft für Chirurgie. Chirurg 88:867-870. https://doi.org/10.1007/ s00104-017-0506-0

18. Mohammed EA, Abdelkader SI, Mohmoud AE (2018cr) Effective role of ascorbic acid as an alternative treatment of methemoglobinemia: a case report. Int J Case Rep Images 9:1. https://doi. org/10.5348/100941z01em2018cr

19. Natanson C, Kern SJ, Lurie P et al (2008) Cellfree hemoglobin-based blood substitutes and risk of myocardial infarction and death. JAMA 299:2304-2312. https://doi.org/10.1001/jama. 300.11.1297-a

20. Posluszny JA, Napolitano LM (2016) Hemoglobinbased oxygen carrier for traumatic hemorrhagic shock treatment in a Jehovah's witness. Arch Trauma Res 5:11-13. https://doi.org/10.5812/atr. 30610

21. Rasanayagam SR, Cooper GM (1996) Two cases of severe postpartum anaemia in Jehovah's witnesses. Int J Obstet Anesth 5:202-205. https:// doi.org/10.1016/S0959-289X(96)80033-1

22. Schälte G, Janz H, Busse J et al (2005) Lifethreatening postoperative blood loss in a Jehovah's witness, treated with high-dose erythropoietin. Br J Anaesth 94:442-444. https://doi.org/10.1093/ bja/aei068

23. Simmons L, Stephenson-Famy A, Easterling T (2012) Optimization of oxygen delivery in a Jehovah's witness with severe anaemia after postpartum haemorrhage. Obstet Med 5:39-41. https://doi.org/10.1258/om.2011.110043

24. Thenuwara K, Thomas J, Ibsen M et al (2017) Use of hyperbaric oxygen therapy and PEGylated carboxyhemoglobin bovine in a Jehovah's witness with life-threatening anemia following postpartum hemorrhage. Int J Obstet Anesth 29:73-80. https://doi.org/10.1016/j.ijoa.2016.10.006

25. Weiskopf RB, Silverman TA (2013) Balancing potential risks and benefits of hemoglobinbased oxygen carriers. Transfusion 53:2327-2333. https://doi.org/10.1111/trf.12339

26. Whitehouse T, Snelson C, Grounds M et al (2014) Intensive care society review of best practice for analgesia and sedation in the critical care

27. Van Wolfswinkel ME, Zwart JJ, Schutte JM et al (2009) Maternal mortality and serious maternal morbidity in Jehovah's witnesses in the Netherlands. Br J Obstet Gynaecol 116:1103-1108. https://doi.org/10.1111/j.1471-0528.2009.02191. $x$

28. Yeykal JM, Stausmire JM, Ahmed MY, Pai A (2014) Right hemicolectomy in a severely anemic Jehovah's witness patient with an extremely low preoperative hemoglobin level and the decision to operate. J Am Osteopath Assoc 114:930-935. https://doi.org/10.7556/jaoa.2014.180

29. Verband Forschender Arzneimittelhersteller e.V. (2020) Behandlung mit Medikamenten, die noch nicht zugelassen sind. https://www.vfa.de/print/ de/patienten/artikel-patienten/behandlungmit-medikamenten-die-noch-nicht-zugelassensind.html.Zugegriffen: 24. Mai 2020

30. Bundesinstitut für Arzneimittel und $\mathrm{Me}$ dizinprodukte (2017) Leitfaden zur Anzeige eines Arzneimittel-Härtefallprogramms nach Arzneimittel-Härtefall-Verordnung (AMHV). https://www.bfarm.de/SharedDocs/ Downloads/DE/Arzneimittel/Zulassung/klinpr/compUse/BfArM-LeitfadenZuArzneimittelHaertefallprogrammen.pdf?_blob= publicationFile\&v=8. Zugegriffen: 24. Mai 2020

\section{Telemedizin}

Marx, Gernot, Rossaint, Rolf, Marx, Nikolaus (Hrsg.)

2021, XVI, 502 S. 98 Abb., 79,99 EUR ISBN 978-3-662-60610-0

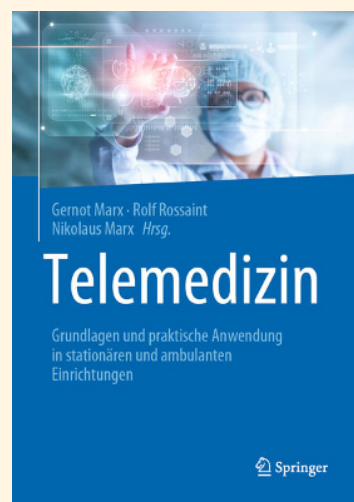

Grundlagen und praktische Anwendung in stationären und ambulanten Einrichtungen:

- Chancen, Risiken und rechtliche Besonderheiten der Telemedizin

- Mit zahlreichen Anwendungsbeispielen

- Mit Blick in die EU und Nachbarländer

Das Buch gibt einen umfassenden Überblick über den aktuellen Stand und die Entwicklungen im Bereich der Telemedizin in Deutschland. Das Herausgeberteam aus einem der führenden Telemedizinzentren Deutschlands und ein interdisziplinäres Autorenteam liefern wesentliche Grundlagen sowie zahlreiche Anwendungsbeispiele aus dem stationären und ambulanten Bereich. Zudem werden Chancen und Risiken der Telemedizin erörtert, so dass der Leser gut für die Zukunft in Klinik oder Praxis gerüstet ist. Ein Grundlagen- und Nachschlagewerk für alle Ärzte, die sich für digitale Zukunft im Gesundheitswesen wappnen und ihre Patienten optimal versorgen möchten. 
Hier steht eine Anzeige.

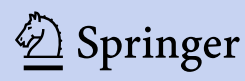

